\title{
Vibrational Spectral Studies of Pure and Doped TGSP Crystals
}

\author{
N. Kartheeswari and K. Viswanathan \\ Department of Physics, Karpagam University, Tamilnadu, Coimbatore 641021, India \\ Correspondence should be addressed to K. Viswanathan; kvnooty@gmail.com
}

Received 21 January 2013; Revised 25 March 2013; Accepted 11 April 2013

Academic Editor: Lahcen Bih

Copyright (c) 2013 N. Kartheeswari and K. Viswanathan. This is an open access article distributed under the Creative Commons Attribution License, which permits unrestricted use, distribution, and reproduction in any medium, provided the original work is properly cited.

\begin{abstract}
Triglycine sulfate crystals (TGS) are an important class of ferroelectric materials. TGS have attracted many researches because of thier room temperature ferroelectric nature. TGS found wide applications in electronic and optical fields. In present work, pure and ADP-, KDP- $(0.2 \mathrm{~mol})$ doped TGSP crystals are grown from solution growth method. Grown crystals are subjected to UV-Vis, IR, and Raman spectral studies. Crystal structure of grown crystals is obtained from powder XRD pattern. Ferroelectric nature of grown crystals is tested using homemade Sawyer-Tower circuit. Electrical conductivity measurements are carried out for pure and doped TGSP crystals.
\end{abstract}

\section{Introduction}

Triglycine sulfate is a ferroelectric material and belongs to the pyroelectric family. It plays a major role in FT-IR instrumentation and infrared detector. It exhibits orderdisorder phase transition at the Curie point $49^{\circ} \mathrm{C}$. The crystal structure of TGS was reported by Hoshino et al. [1]. The unit cell of TGS contains three types of glycines GI, GII, and GIII. Glycine I is in the form of zwitterion. GII and GIII are two planar glycines. They have protonated carboxyl groups which have taken protons from the sulfuric acid. They form chain-like system with $\mathrm{SO}_{4}{ }^{2-}$ group. The neighbouring chains are connected by $\mathrm{SO}_{4}{ }^{2-}$ groups of adjacent chains and GI group. Such configuration of TGS is regarded as particularly important for the ferroelectric behavior of TGS crystal. The orientation of positively charged $\mathrm{NH}_{3}$ groups determines the direction of spontaneous polarization in TGS. The spontaneous polarization reversal in TGS is due to the proton transfer between glycine and glycinium ions [2]. TGS exhibits order-disorder phase transition at the Curie point. Above Curie temperature, it is in the Paraelectric phase with space group symmetry $\mathrm{P} 2{ }_{1} / \mathrm{m}$ and below Curie it is in the ferroelectric phase with space group symmetry $\mathrm{P} 2{ }_{1}$ with two formula units per unit cell [3]. The unit cell parameters of TGS are $a=9.15 \AA, b=12.6445 \AA, c=5.725 \AA$, and $\beta=105.53^{\circ}$ [4]. In TGS, majority of domains were found in the form of rod shapped with lenticular cross-sections elongated in the direction perpendicular to $c$-axis [5]. TGS provides rich lattice vibration spectrum below $300 \mathrm{~cm}^{-1}$ [6]. The main disadvantage of TGS is its lower Curie temperature and easy depolarization by time, electrical, mechanical, and thermal means. Because glycine has no asymmetric carbon and it is optically inactive, it is believed that doping of TGS with an optically active molecule will keep permanent polarization in TGS lattice. There are a large number of researches going on TGS to minimize the depolarization effect and to keep permanent polarization in TGS lattice. There is no change in crystal structure for Nd-doped TGS and it is reported that $\mathrm{Nd}$ is coordinated with glycine [2]. But in the case of ADP, L-Asparagine- and L-tryptophan-doped samples' change in crystal morphology is observed [7-9]. $T_{c}$ value is higher for 1-threonine-, dl-threonine-, l-methionine-doped crystals and TGSP crystals $[10,11]$. Spectral investigation of TGS doped with ADP, L-tryptophan, phosphoric acid, L-lysine, L-cystine reveals that doped samples have lower spontaneous polarization values $P_{s}$ and higher coercive field values compared to pure TGS. Higher coercive field value implies that the crystal is in the monodomain state $[7,9,11-13]$. Pyroelectric 


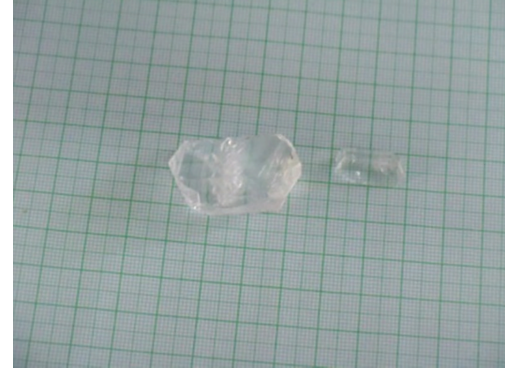

TGSP

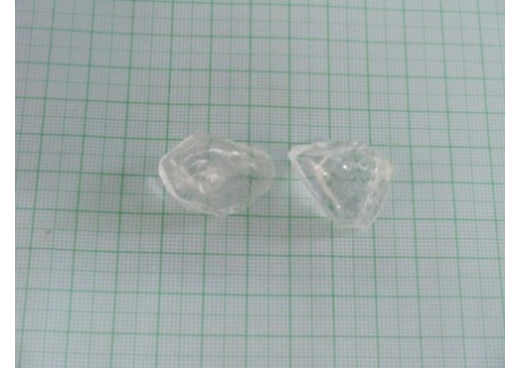

TGSP + ADP

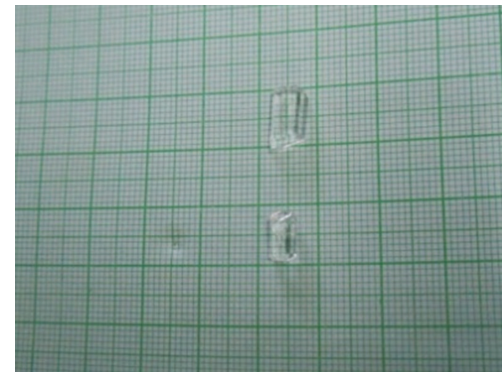

TGSP + KDP

FIGURE 1: Grown crystals of pure and ADP-, KDP- (0.2 mol) doped TGSP.

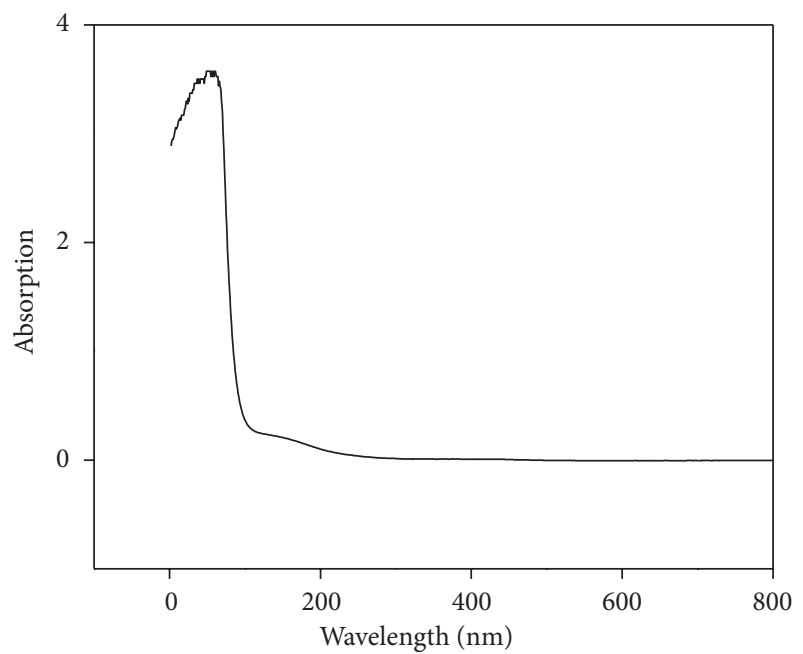

(a)

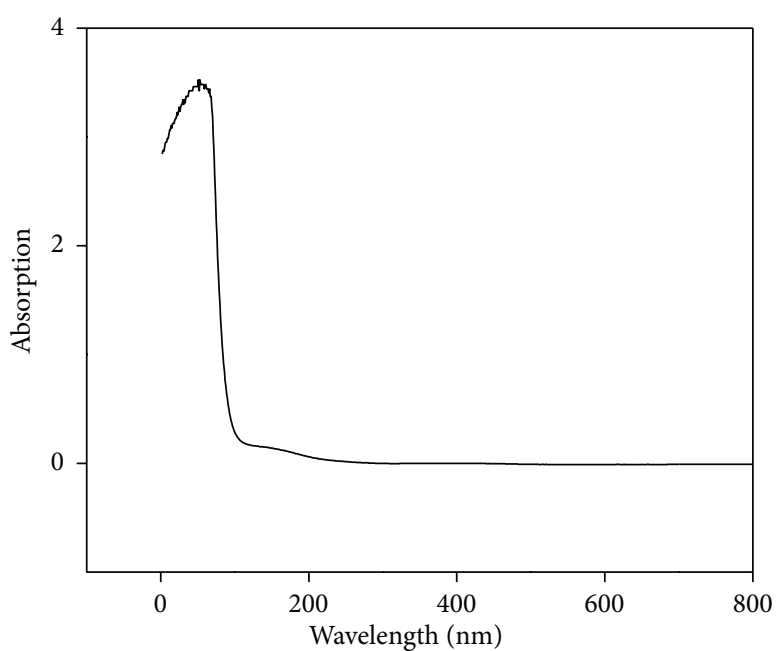

(b)

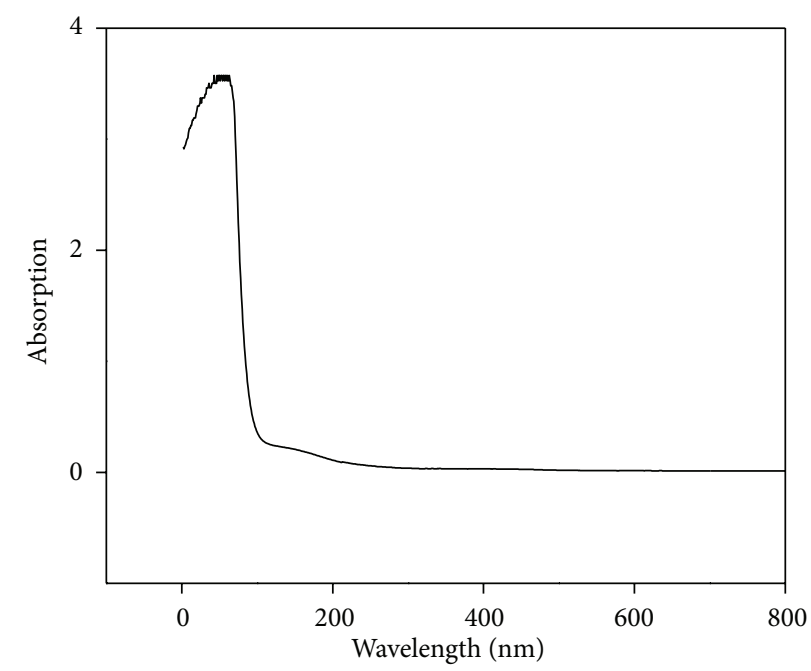

(c)

Figure 2: UV-Vis absorption spectra of (a) TGSP, (b) TGSP + ADP, and (c) TGSP + KDP crystals. 


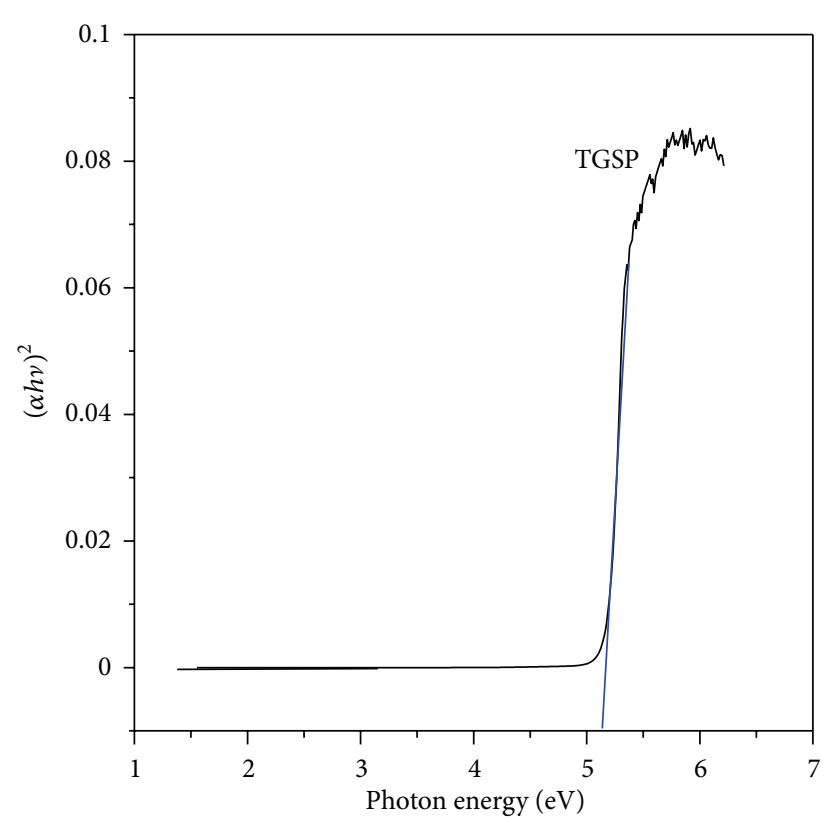

(a)

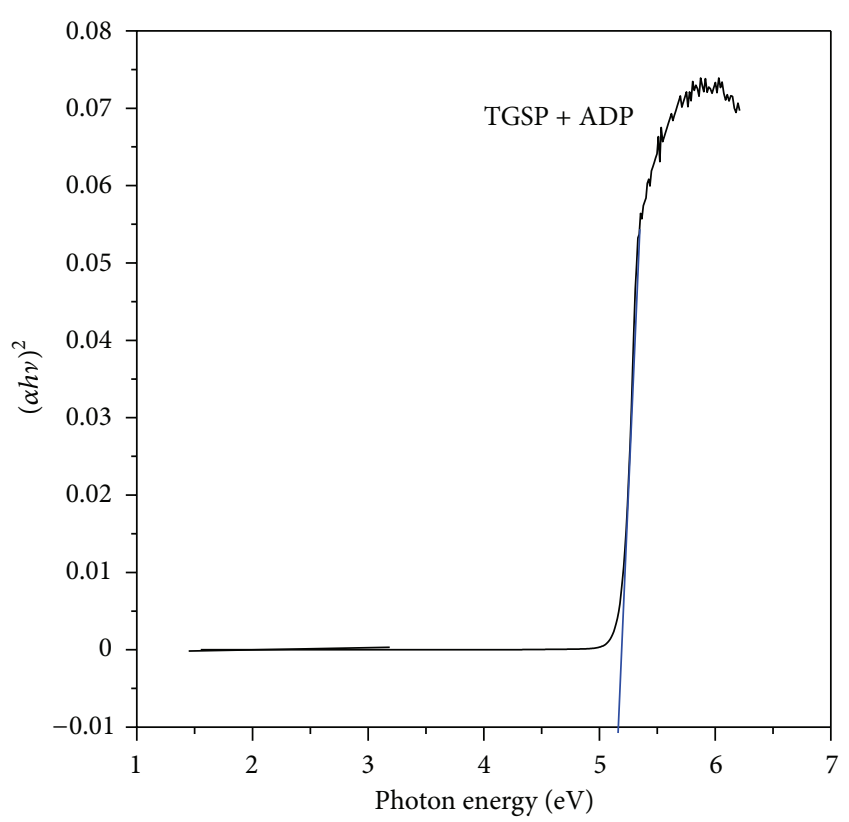

(b)

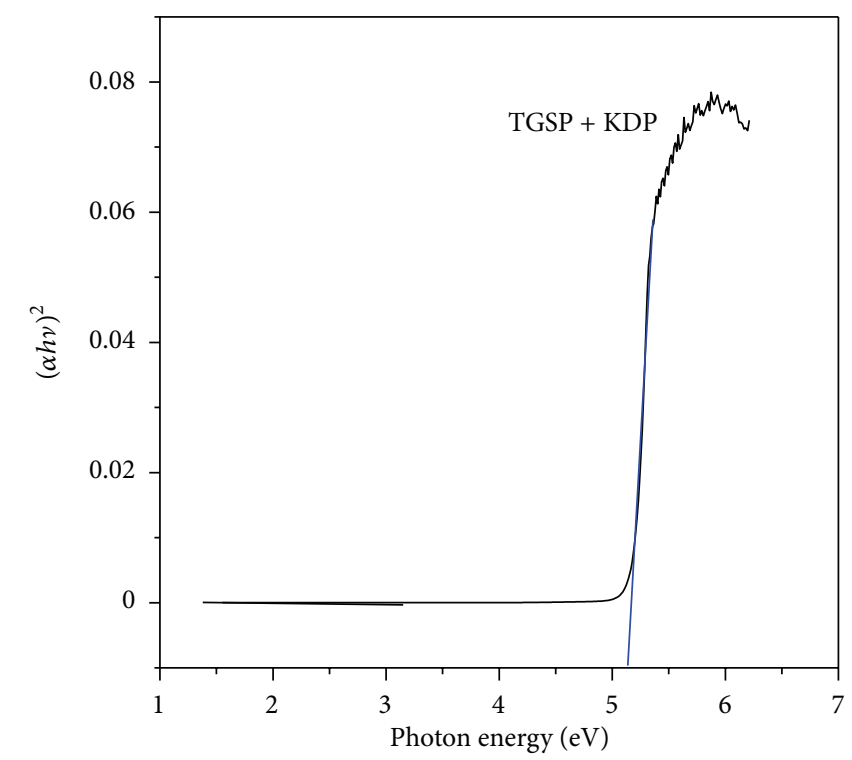

(c)

FIGURE 3: Urbach plot of (a) TGSP, (b) TGSP + ADP, and (c) TGSP + KDP crystals.

coefficient is increased for thiourea-, L-alanine- and DLalanine-, L-lysine-doped samples. [14-16]. Incorporation of nitric acid and EDTA into TGS crystal increases the dielectric constant value $[17,18]$. The substitution of amino acids 1threonine, dl-threonine, l-methionine, and L-cystine results in the decrease of dielectric constant value compared to pure TGS crystal $[10,13]$. Zinc chloride- and urea-doped samples exhibit nonlinear optical property and have SHG efficiency very much greater than standard KDP crystal [19, 20]. In case of LGLM, La-, Ce-, Nd-, L-lysine-doped TGS crystal strong internal bias field is created, indicating that the dopant reduces the depolarizing effects in TGS crystals.
The internal bias field fixes the polarization in a preferential direction with minimum possibility of polarization reversal $[16,21,22]$. Doping of TGS with metal ions $\mathrm{Fe}^{3+}, \mathrm{Cr}^{3+}$, and $\mathrm{Co}^{2+}$ decreased the indirect band gap [23]. Investigation of TGS samples previously influenced by electric field $E$ perpendicular to ferroelectric $b$-axis reveals that there are rigid stripped domains parallel to $c$-axis [24]. Vickers' microhardness study reveals that hardness value is increased for TGS when doping with L-lysine and imino diacetic acid [25, 26]. But hardness value of crystal is decreased for doping TGS with 1-threonine, dl-threonine and l-methionine, L-cystine, thiourea, and EDTA $[10,13,14,18]$. In present work, pure and 


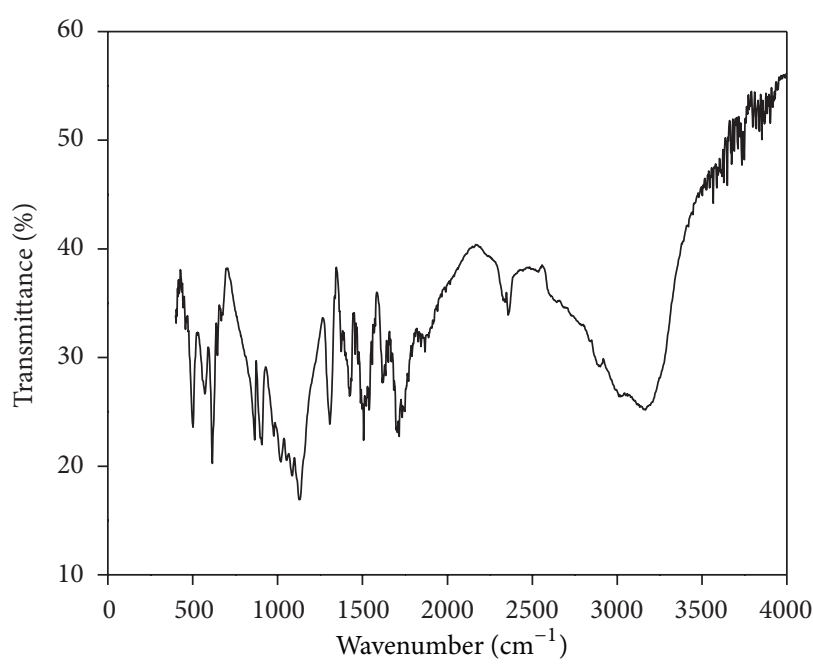

(a)

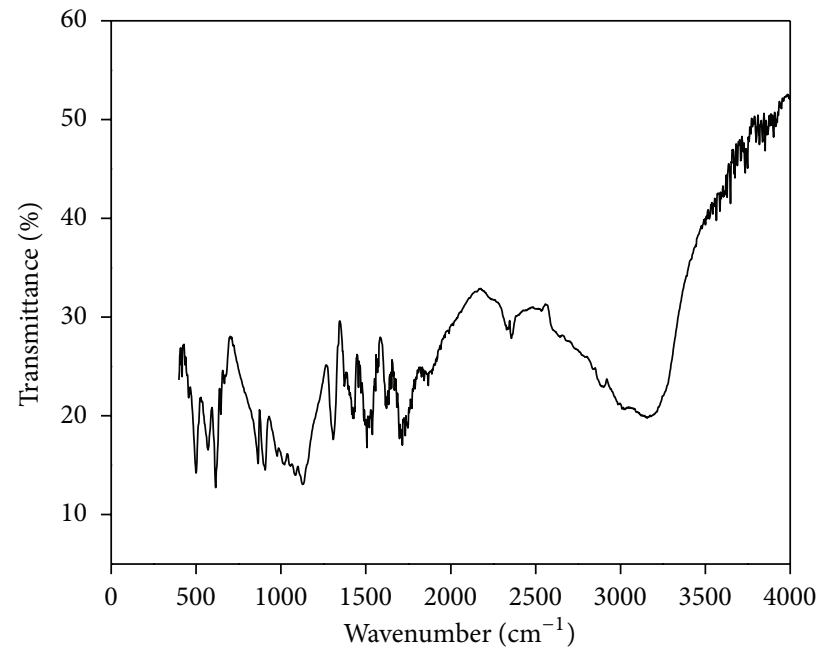

(b)

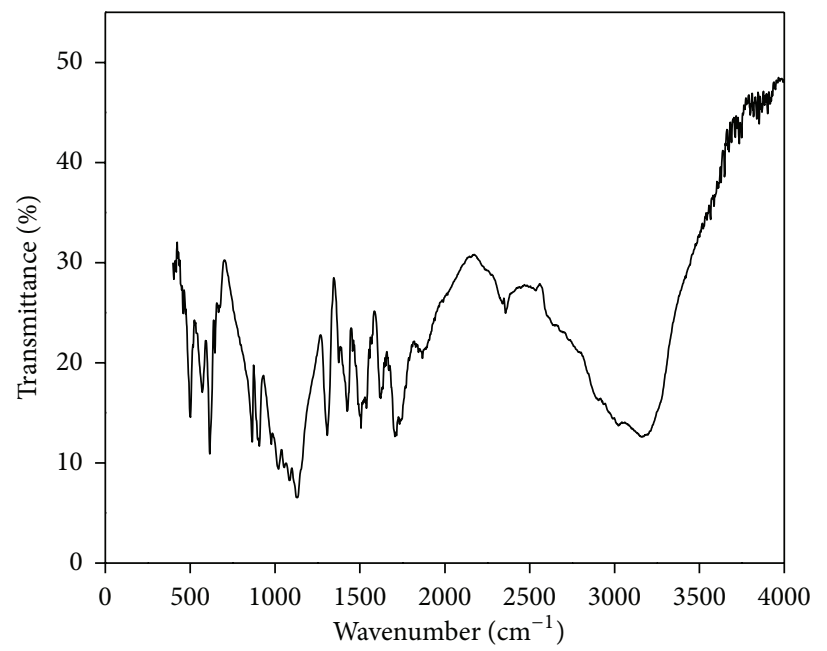

(c)

Figure 4: FT-IR spectra of (a) TGSP, (b) TGSP + ADP, and (c) TGSP + KDP crystals.

TABLE 1: Energy band gap values of grown crystals.

\begin{tabular}{lcc}
\hline Crystal & $\begin{array}{c}\text { Eg (calculated from } \\
\left.\mathrm{Eg}=1240 / \lambda_{\max } \mathrm{eV}\right) \mathrm{eV}\end{array}$ & $\begin{array}{c}\text { Eg (obtained from } \\
\text { Urbach Plot) eV }\end{array}$ \\
\hline TGSP & 5.34 & 5.1496 \\
TGSP + ADP & 5.32 & 5.1606 \\
TGSP + KDP & 5.3 & 5.1496 \\
\hline
\end{tabular}

ADP-, KDP- (0.2 mol) doped TGSP crystals are grown from solution.

\section{Crystal Growth and Characterization}

Pure and ADP-, KDP- $(0.2 \mathrm{~mol})$ doped TGSP crystals were grown by solution growth method [4]. Pure TGSP crystal is grown by taking 3 moles of Analar grade Glycine, $0.5 \mathrm{~mol}$ of sulphuric acid, and $0.5 \mathrm{~mol}$ of phosphoric acid $100 \mathrm{~mL}$ of distilled water. The solution is stirred well for $3 \mathrm{hrs}$. Then the solution is filtered and left to slow evaporation. ADP- and KDP- $(0.2 \mathrm{~mol})$ doped crystals are grown by the same method. Good-quality crystals are obtained within the growth period of 1-3 months. Grown crystals are shown in Figure 1. UVVis absorption spectra of grown crystals are recorded in the wavelength range from 200 to $800 \mathrm{~nm}$ using UV-Vis 2450 Make Shimadzu model spectrophotometer. FT-IR spectra are recorded in the frequency range from 400 to $4000 \mathrm{~cm}^{-1}$ using IR Affinity Make Shimadzu model spectrophotometer. FTRaman spectra were recorded in the frequency range from 0 to $3500 \mathrm{~cm}^{-1}$ using the excitation radiation of $5145 \mathrm{~A}^{\circ}$ using Lab Ram HR 800 model spectrophotometer. Ferroelectric hysteresis study was carried out using homemade SawyerTower circuit [27].

\section{Results and Discussion}

3.1. UV-Vis Spectral Investigation of Grown Crystals. Figure 2 shows UV-Vis absorption spectra of pure and ADP-, KDP- 
TABLE 2: FT-IR analysis.

\begin{tabular}{|c|c|c|c|}
\hline Pure TGSP & TGSP + ADP & TGSP + KDP & Assignment \\
\hline $\begin{array}{l}401 \mathrm{w}-472 \mathrm{w} \\
\text { (8 lines) }\end{array}$ & $\begin{array}{l}412 \mathrm{w}-455 \mathrm{w} \\
(5 \text { lines })\end{array}$ & $\begin{array}{l}403 \mathrm{w}-472 \mathrm{w} \\
(7 \text { lines })\end{array}$ & $v\left(\mathrm{PO}_{4}\right), v\left(\mathrm{SO}_{4}\right)$ \\
\hline $503 \mathrm{w}$ & $\begin{array}{l}501 \mathrm{w}-536 \mathrm{w} \\
\text { (4 lines) }\end{array}$ & $501 \mathrm{w}$ & $\tau(\mathrm{C}-\mathrm{N})$ \\
\hline $572 \mathrm{w}$ & $571 \mathrm{w}$ & $569 \mathrm{w}$ & $v\left(\mathrm{SO}_{4}^{-}\right)$ \\
\hline $\begin{array}{l}615 \mathrm{~m} \\
646 \mathrm{w}\end{array}$ & $\begin{array}{l}615 \mathrm{~m}-646 \mathrm{w} \\
(3 \text { lines })\end{array}$ & $\begin{array}{l}615 \mathrm{~m} \\
646 \mathrm{w}\end{array}$ & $\begin{array}{c}\delta(\mathrm{C}-\mathrm{N}), \mathrm{NH}_{3}^{+} \\
\quad \text { oscillation }\end{array}$ \\
\hline $\begin{array}{l}669 \mathrm{~m} \\
678 \mathrm{w}\end{array}$ & $\begin{array}{l}667 \mathrm{~m} \\
680 \mathrm{w}\end{array}$ & $\begin{array}{l}669 \mathrm{~m} \\
678 \mathrm{w}\end{array}$ & $\nu(\mathrm{S}-\mathrm{H})$ \\
\hline $\begin{array}{l}866 \mathrm{~m} \\
898 \mathrm{~m}\end{array}$ & $866 \mathrm{~m}$ & $866 \mathrm{~m}$ & $\nu(\mathrm{C}-\mathrm{C})$ \\
\hline $908 \mathrm{w}$ & $908 \mathrm{w}$ & $906 \mathrm{w}$ & $\nu(\mathrm{P}-\mathrm{O}-\mathrm{H}), \rho\left(\mathrm{NH}_{3}\right)$ \\
\hline 979 s & $979 \mathrm{~s}$ & $978 \mathrm{~s}$ & $v(\mathrm{C}-\mathrm{C}-\mathrm{N}), v\left(\mathrm{SO}_{4}^{-}\right)$ \\
\hline $1018 \mathrm{w}$ & - & $1018 \mathrm{w}$ & $\begin{array}{c}\omega\left(\mathrm{CH}_{2}\right), \delta(\mathrm{C}-\mathrm{C}) \\
\nu(\mathrm{C}-\mathrm{N})\end{array}$ \\
\hline $1051 \mathrm{w}$ & $1051 \mathrm{w}$ & $1051 \mathrm{w}$ & $v(\mathrm{P}-\mathrm{O}-\mathrm{H})$ \\
\hline $1083 \mathrm{w}$ & $1083 \mathrm{w}$ & $1082 \mathrm{w}$ & $v(\mathrm{P}-\mathrm{O}-\mathrm{H}), v(\mathrm{C}=\mathrm{O})$ \\
\hline $\begin{array}{l}1111 \mathrm{~m} \\
1124 \mathrm{~s}\end{array}$ & $1109 \mathrm{~m}$ & $\begin{array}{l}1109 \mathrm{~m} \\
1130 \mathrm{~s}\end{array}$ & $\rho\left(\mathrm{CH}_{2}\right), \nu(\mathrm{C}-\mathrm{C}), \nu\left(\mathrm{SO}_{4}\right)$ \\
\hline $1309 \mathrm{~m}$ & $1309 \mathrm{~m}$ & $1309 \mathrm{~m}$ & $\begin{array}{c}\delta\left(\mathrm{CH}_{2}\right) \text { of glycine } \\
\nu(\mathrm{C}-\mathrm{C})\end{array}$ \\
\hline $1338 \mathrm{w}$ & $1338 \mathrm{w}$ & - & $v\left(\mathrm{NO}_{2}\right)$ \\
\hline $1363 \mathrm{w}$ & $1363 \mathrm{w}$ & - & $v(\mathrm{C}-\mathrm{N})$ \\
\hline $1375 \mathrm{~m}$ & $1375 \mathrm{~m}$ & $1377 \mathrm{~m}$ & $\delta\left(\mathrm{CH}_{2}\right)$ \\
\hline $1386 \mathrm{w}$ & $1386 \mathrm{w}$ & $1388 \mathrm{w}$ & $v\left(\mathrm{NH}_{4}\right)$ \\
\hline $1398 \mathrm{w}$ & $1396 \mathrm{w}$ & - & $\nu(\mathrm{C}=\mathrm{O}), \nu\left(\mathrm{NH}_{4}\right)$ \\
\hline $1404 \mathrm{w}$ & 1404 & - & $\delta\left(\mathrm{NH}_{4}\right)$ \\
\hline $1409 \mathrm{w}$ & - & - & $v_{\mathrm{as}}\left(\mathrm{COO}^{-}\right), \delta\left(\mathrm{NH}_{4}\right)$ \\
\hline $\begin{array}{l}1417 \mathrm{~m} \\
1425 \mathrm{~m}\end{array}$ & $\begin{array}{l}1417 \mathrm{~m} \\
1423 \mathrm{~m}\end{array}$ & $\begin{array}{l}1421 \mathrm{~m} \\
1427 \mathrm{~m}\end{array}$ & $\nu\left(\mathrm{COO}^{-}\right), \delta\left(\mathrm{CH}_{2}\right)$ \\
\hline $\begin{array}{l}1435 \mathrm{w} \\
1448 \mathrm{w}\end{array}$ & $1435 \mathrm{w}$ & $1435 \mathrm{w}$ & $\delta\left(\mathrm{NH}_{3}\right)$ \\
\hline $\begin{array}{l}1456 \mathrm{~m}-1498 \mathrm{w} \\
(5 \text { lines) }\end{array}$ & $\begin{array}{l}1456 \mathrm{~m}-1496 \mathrm{w} \\
\quad(5 \text { lines })\end{array}$ & $\begin{array}{l}1458 \mathrm{~m}-1490 \mathrm{w} \\
\quad(3 \text { lines })\end{array}$ & $v\left(\mathrm{NH}_{4}\right), v\left(\mathrm{COO}^{-}\right)$ \\
\hline $\begin{array}{l}1506 \mathrm{~m}-1558 \mathrm{w} \\
(5 \text { lines })\end{array}$ & $\begin{array}{l}1506 \mathrm{~m}-1558 \mathrm{w} \\
\quad(7 \text { lines })\end{array}$ & $\begin{array}{l}1506 \mathrm{~m}-1558 \mathrm{w} \\
\quad(4 \text { lines })\end{array}$ & $\delta\left(\mathrm{NH}_{3}\right)$ \\
\hline $\begin{array}{l}1570 \mathrm{~m}-1595 \mathrm{w} \\
\text { (3 lines) }\end{array}$ & $\begin{array}{l}1568 \mathrm{~m} \\
1575 \mathrm{w}\end{array}$ & $\begin{array}{l}1570 \mathrm{~m} \\
1575 \mathrm{w}\end{array}$ & $v\left(\mathrm{COO}^{-}\right)$ \\
\hline $\begin{array}{l}1616 \mathrm{w} \\
1622 \mathrm{w}\end{array}$ & $\begin{array}{l}1616 \mathrm{w} \\
1622 \mathrm{w}\end{array}$ & $1622 \mathrm{w}$ & $v_{\mathrm{as}}\left(\mathrm{COO}^{-}\right), \delta\left(\mathrm{NH}_{3}\right)$ \\
\hline $\begin{array}{l}1635 w \\
1647 w \\
1653 w\end{array}$ & $\begin{array}{l}1633 \mathrm{w} \\
1645 \mathrm{w} \\
1653 \mathrm{w}\end{array}$ & $\begin{array}{l}1635 w \\
1647 w \\
1653 w\end{array}$ & $v(\mathrm{O}=\mathrm{P}-\mathrm{OH}), \nu(\mathrm{C}=\mathrm{C})$ \\
\hline $\begin{array}{l}1662 \mathrm{w} \\
1670 \mathrm{w}\end{array}$ & $\begin{array}{l}1662 \mathrm{w}-1674 \mathrm{w} \\
\quad(3 \text { lines })\end{array}$ & $\begin{array}{l}1662 \mathrm{w} \\
1672 \mathrm{w}\end{array}$ & $\delta\left(\mathrm{H}_{2} \mathrm{O}\right), \nu(\mathrm{C}=\mathrm{C})$ \\
\hline $\begin{array}{l}1683 \mathrm{w}-1869 \mathrm{w} \\
(12 \text { lines) }\end{array}$ & $\begin{array}{l}1683 \mathrm{w}-1867 \mathrm{w} \\
(19 \text { lines })\end{array}$ & $\begin{array}{l}1685 \mathrm{w}-1869 \mathrm{w} \\
\text { (12 lines) }\end{array}$ & $\nu(\mathrm{C}=\mathrm{O}), v\left(\mathrm{C}=\mathrm{NH}_{4}\right)$ \\
\hline $\begin{array}{l}1942 \mathrm{w}-2360 \mathrm{w} \\
(5 \text { lines })\end{array}$ & $\begin{array}{l}1942 \mathrm{w}-2358 \mathrm{w} \\
\quad(4 \text { lines })\end{array}$ & $\begin{array}{l}2017 \mathrm{w} \\
2357 \mathrm{w}\end{array}$ & $\begin{array}{c}\text { Overtone and } \\
\text { combination bands }\end{array}$ \\
\hline
\end{tabular}


TABLE 2: Continued.

\begin{tabular}{|c|c|c|c|}
\hline Pure TGSP & TGSP + ADP & $\mathrm{TGSP}+\mathrm{KDP}$ & Assignment \\
\hline $\begin{array}{l}3502 \mathrm{w}-3547 \mathrm{w} \\
\text { (5 lines) }\end{array}$ & $\begin{array}{c}3502 \mathrm{w}-3545 \mathrm{w} \\
\quad(5 \text { lines })\end{array}$ & $\begin{array}{c}3502 \mathrm{w}-3545 \mathrm{w} \\
\quad(4 \text { lines })\end{array}$ & $v(\mathrm{O}-\mathrm{H}), v(\mathrm{~N}-\mathrm{H})$ \\
\hline $\begin{array}{l}3566 \mathrm{w}-3701 \mathrm{w} \\
(11 \text { lines) }\end{array}$ & $\begin{array}{l}3550 \mathrm{w}-3701 \mathrm{w} \\
(13 \text { lines })\end{array}$ & $\begin{array}{c}3566 \mathrm{w}-3701 \mathrm{w} \\
\quad(9 \text { lines })\end{array}$ & $\begin{array}{c}\nu(\mathrm{O}-\mathrm{H}), \nu(\mathrm{C}-\mathrm{H}) \\
\nu(\mathrm{C}=\mathrm{O}) \\
\delta(\mathrm{P}-\mathrm{O}-\mathrm{H})\end{array}$ \\
\hline $\begin{array}{l}3711 \mathrm{w}-3947 \mathrm{w} \\
(24 \text { lines) }\end{array}$ & $\begin{array}{c}3711 \mathrm{w}-3961 \mathrm{w} \\
(24 \text { lines })\end{array}$ & $\begin{array}{c}3711 \mathrm{w}-3963 \mathrm{w} \\
(25 \text { lines })\end{array}$ & $v(\mathrm{O}-\mathrm{H})$ of water \\
\hline
\end{tabular}

$\nu_{\mathrm{s}}$ : symmetric stretching, $\nu_{\mathrm{as}}$ : asymmetric stretching, $\delta$ : bending, $\rho$ : rocking, $\omega$ : wagging, and $\tau$ : torsional vibrations.

TABLE 3: FT-RAMAN analysis.

\begin{tabular}{|c|c|c|c|}
\hline Pure TGSP & TGSP + ADP & TGSP + KDP & Assignment \\
\hline $\begin{array}{l}74 \mathrm{~m} \\
103 \mathrm{~m}\end{array}$ & $\begin{array}{l}74 \mathrm{~m}-340 \mathrm{~m} \\
(4 \text { lines })\end{array}$ & $\begin{array}{l}73 \mathrm{~m}-234 \mathrm{~m} \\
\quad(5 \text { lines })\end{array}$ & Lattice-mode vibration of glycine \\
\hline- & $466 \mathrm{~m}$ & $451 \mathrm{~m}$ & Lattice-mode vibration of $\mathrm{SO}_{4}, \delta(\mathrm{C}-\mathrm{N})$ out of plane, $\nu\left(\mathrm{PO}_{4}\right)$ \\
\hline- & $\begin{array}{l}506 \mathrm{w}-590 \mathrm{w} \\
\quad(3 \text { lines })\end{array}$ & $\begin{array}{l}506 \mathrm{w} \\
590 \mathrm{w}\end{array}$ & $\tau(\mathrm{C}-\mathrm{N}), v\left(\mathrm{PO}_{4}\right), \delta(\mathrm{C}-\mathrm{CO}), v\left(\mathrm{SO}_{4}^{-}\right)$ \\
\hline $\begin{array}{l}632 \mathrm{w} \\
633 \mathrm{w}\end{array}$ & $614 \mathrm{w}$ & $628 \mathrm{w}$ & $v\left(\mathrm{SO}_{4}\right), \delta(\mathrm{C}-\mathrm{N})$ in plane, $\mathrm{NH}_{3}{ }^{+}$oscillation \\
\hline- & $678 \mathrm{~m}$ & $677 \mathrm{~m}$ & $v(\mathrm{C}-\mathrm{C})$ \\
\hline $869 \mathrm{~m}$ & $871 \mathrm{~m}$ & - & $\nu(\mathrm{C}-\mathrm{C})$ \\
\hline $889 \mathrm{w}$ & $890 \mathrm{w}$ & $890 \mathrm{w}$ & $\rho\left(\mathrm{CH}_{2}\right), \rho\left(\mathrm{NH}_{3}\right), v(\mathrm{C}-\mathrm{C}), v(\mathrm{P}-\mathrm{O}-\mathrm{H})$ \\
\hline $978 \mathrm{~s}$ & $978 \mathrm{~s}$ & $978 \mathrm{~s}$ & $v(\mathrm{C}-\mathrm{C}-\mathrm{N}), v\left(\mathrm{SO}_{4}^{-}\right)$ \\
\hline $1051 \mathrm{w}$ & $1042 \mathrm{w}$ & $1045 \mathrm{w}$ & $\omega\left(\mathrm{CH}_{2}\right), \delta(\mathrm{C}-\mathrm{C}), \nu(\mathrm{C}-\mathrm{N}), \nu(\mathrm{C}-\mathrm{N}-\mathrm{H}), \nu(\mathrm{P}-\mathrm{O}-\mathrm{H})$ \\
\hline $\begin{array}{l}1114 \mathrm{w} \\
1122 \mathrm{w}\end{array}$ & $\begin{array}{l}1123 \mathrm{w} \\
1128 \mathrm{w}\end{array}$ & $\begin{array}{l}1124 \mathrm{w} \\
1157 \mathrm{w}\end{array}$ & $\rho\left(\mathrm{CH}_{2}\right), v(\mathrm{C}-\mathrm{C}), v\left(\mathrm{SO}_{4}\right)$ \\
\hline $\begin{array}{l}1304 \mathrm{w} \\
1314 \mathrm{w}\end{array}$ & $1306 \mathrm{w}$ & $1309 \mathrm{w}$ & $\delta(\mathrm{C}-\mathrm{H}), v(\mathrm{C}-\mathrm{C})$ \\
\hline $1414 \mathrm{w}$ & $1416 \mathrm{w}$ & $1413 \mathrm{w}$ & $v_{\mathrm{s}}\left(\mathrm{COO}^{-}\right), \delta\left(\mathrm{CH}_{2}\right)$ \\
\hline 1439 & $\begin{array}{l}1441 \\
1496\end{array}$ & 1443 & $\nu\left(\mathrm{NH}_{4}\right), \delta\left(\mathrm{CH}_{2}\right)$ \\
\hline 1605 & 1607 & 1606 & $v\left(\mathrm{COO}^{-}\right), v\left(\mathrm{NH}_{4}\right), v(\mathrm{P}-\mathrm{O}-\mathrm{H}), v(\mathrm{C}=\mathrm{C})$ \\
\hline $1683 \mathrm{w}$ & 1678 & $\begin{array}{l}1683 \\
1762\end{array}$ & $v(\mathrm{C}=\mathrm{O}), v(\mathrm{O}-\mathrm{H}-\mathrm{O})$ \\
\hline 2648 & - & - & $\delta\left(\mathrm{H}_{2} \mathrm{O}\right), v(\mathrm{COOH})$ \\
\hline 2982 & 2958 & $\begin{array}{l}2958 \\
2982\end{array}$ & $v(\mathrm{C}-\mathrm{H})$ \\
\hline $\begin{array}{l}3014 \mathrm{w} \\
3057 \mathrm{w}\end{array}$ & $\begin{array}{l}3019 w \\
3140 w\end{array}$ & $\begin{array}{l}3017 \mathrm{w} \\
3143 \mathrm{w}\end{array}$ & $\nu\left(\mathrm{NH}_{4}\right)$ \\
\hline
\end{tabular}

$\nu_{\mathrm{s}}$ : symmetric stretching, $\nu_{\text {as }}$ : asymmetric stretching, $\delta$ : bending, $\rho$ : rocking, $\omega$ : wagging, and $\tau$ : torsional vibrations.

TABLE 4: Lattice parameter values of grown crystals.

\begin{tabular}{lcccc}
\hline Crystal & $a(\mathrm{~nm})$ & $b(\mathrm{~nm})$ & $c(\mathrm{~nm})$ & $\beta(\mathrm{deg})$ \\
\hline TGSP & 9.4173 & 12.6449 & 5.7734 & 110.36 \\
TGSP + ADP & 9.5786 & 12.58434 & 5.8198 & 110.36 \\
TGSP + KDP & 9.4566 & 12.67908 & 5.7222 & 110.36 \\
\hline
\end{tabular}

$(0.2 \mathrm{~mol})$ doped TGSP crystals. For TGSP crystal, $\lambda_{\max }=$ $232 \mathrm{~nm}$. For TGSP + ADP, $\lambda_{\max }=233 \mathrm{~nm}$ and for TGSP + $\mathrm{KDP}, \lambda_{\max }=233.5 \mathrm{~nm}$. For doped crystals, there is slight shift in wavelength of maximum absorption $\lambda_{\max }$. This is referred to as bathochromic shift. From this, it is observed that there is change in energy levels to effect transition. For doped samples, the energy required to effect the electron promotion is lesser than that of pure TGSP so that the wavelength that provides this energy is increased for doped crystals. Absorption at lower wavelength reveals that there must be higher energy transition corresponding to $\mathrm{C}=\mathrm{C}-$ $\mathrm{NO}_{2}$ group. It is observed that all these crystals have transmission percentage of above $90 \%$. Energy band gap values were found out using the relation $E=1240 / \lambda_{\max } \mathrm{eV}$ [28]. 


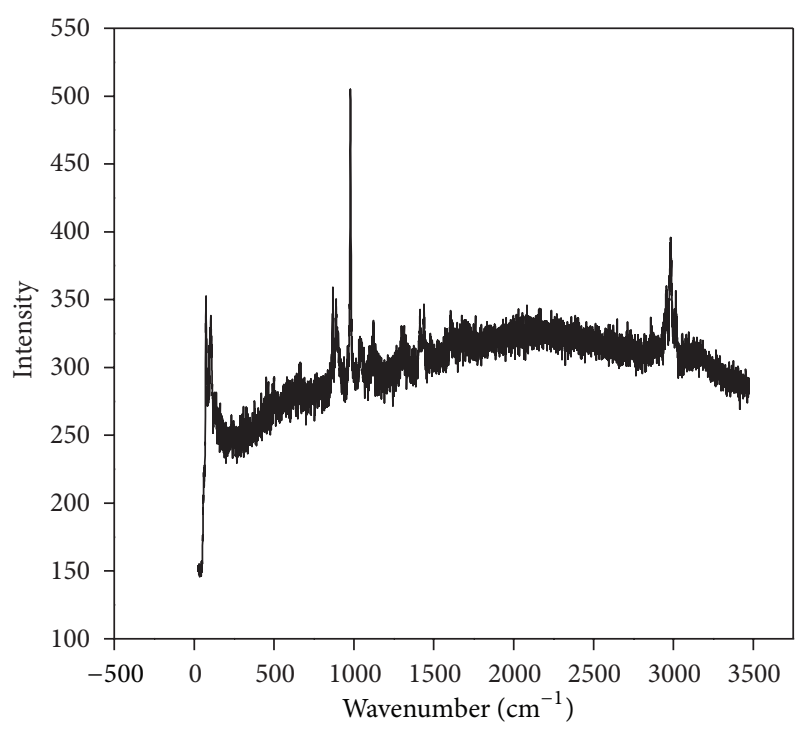

(a)

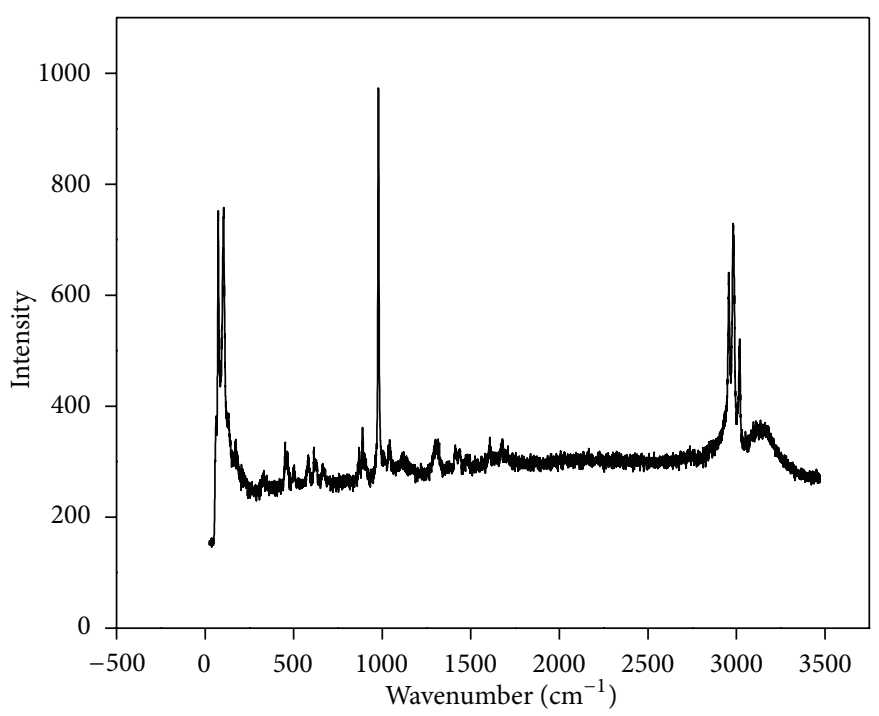

(b)

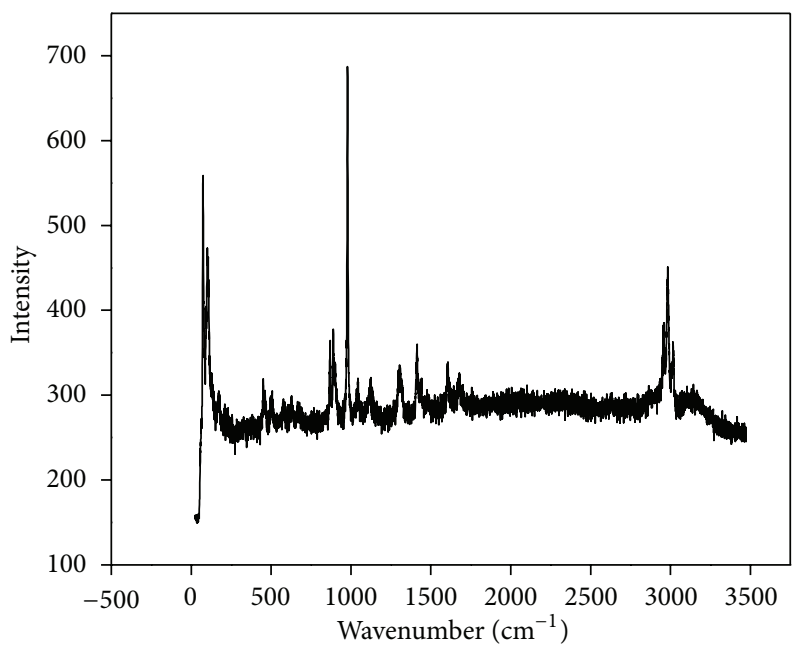

(c)

FIgURE 5: FT-Raman spectra of (a) TGSP (b) TGSP + ADP (c) TGSP + KDP crystals.

TABLE 5: Ferroelectric hysteresis loop measurement values.

\begin{tabular}{lccc}
\hline Crystal & $\begin{array}{c}\text { Spontaneous } \\
\text { polarization } \\
\operatorname{Ps}\left(\mu \mathrm{C} / \mathrm{cm}^{2}\right)\end{array}$ & $\begin{array}{c}\text { Remnant } \\
\text { polarization } \\
\operatorname{Pr}\left(\mu \mathrm{C} / \mathrm{cm}^{2}\right)\end{array}$ & $\begin{array}{c}\text { Coercive field } \\
\text { value } \\
(\mathrm{kV} / \mathrm{cm})\end{array}$ \\
\hline TGSP & 1.712 & 1.04 & 6 \\
TGSP + ADP & 3.1388 & 1.5023 & 6.01 \\
TGSP + KDP & 3.6804 & 1.8731 & 6.02 \\
\hline
\end{tabular}

Also energy band gap values are obtained from Urbach plot and results are shown in Table 1. Figure 3 shows Urbach plots for grown crystals.

3.2. FT-IR Spectral Analysis. Figure 4 shows FT-IR spectra of pure and ADP-, KDP-doped TGSP crystals. FT-IR spectrum of pure TGSP crystal matches very well with the earlier reported values of pure TGS crystal [17]. All expected characteristic vibrations are observed and assignments were tabulated in Table 2. IR bands at $1425 \mathrm{~cm}^{-1}$ and $1622 \mathrm{~cm}^{-1}$ corresponding to symmetric and asymmetric stretching vibrations of $\mathrm{COO}^{-}$indicate the zwitterion configuration of glycine [17]. IR bands observed in the region between $1683 \mathrm{~cm}^{-1}$ to $1869 \mathrm{~cm}^{-1}$ corresponding to stretching vibration of $\mathrm{C}=\mathrm{O}$ indicate the presence of glycinium ion configuration [17]. Degenerate modes of $\mathrm{NH}_{3}$ bending and $\mathrm{C}=\mathrm{O}, \mathrm{NH}_{4}, \mathrm{C}-$ $\mathrm{H}$, and $\mathrm{O}-\mathrm{H}$ stretching vibrations are observed. FT-IR spectra of ADP-, KDP doped samples provide very similar features as those of pure TGSP. More bands were located at the same positions as those of pure TGSP. There is a very slight shift observed in band positions compared to pure TGSP. But doped samples provide less resolution of bands. Some bands are broadened and some are narrowed. Degeneracy is more for doped samples than that of pure TGSP. 


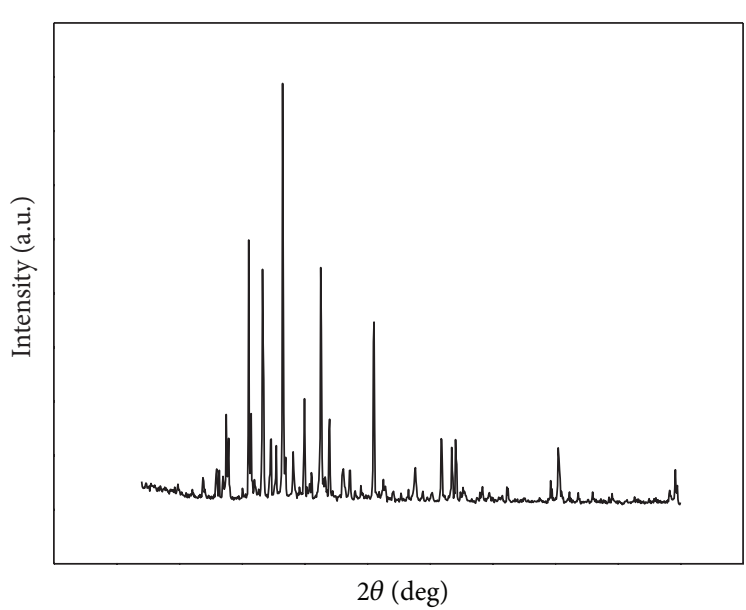

(a)

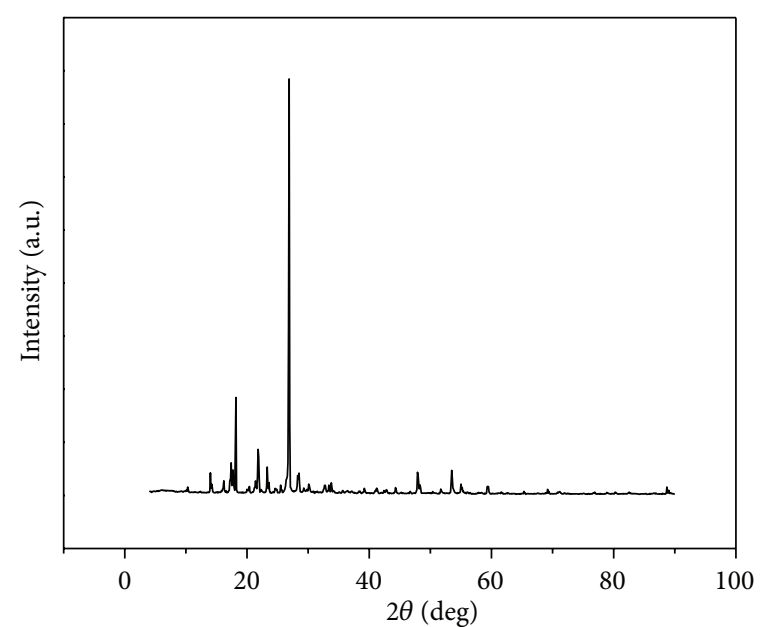

(b)

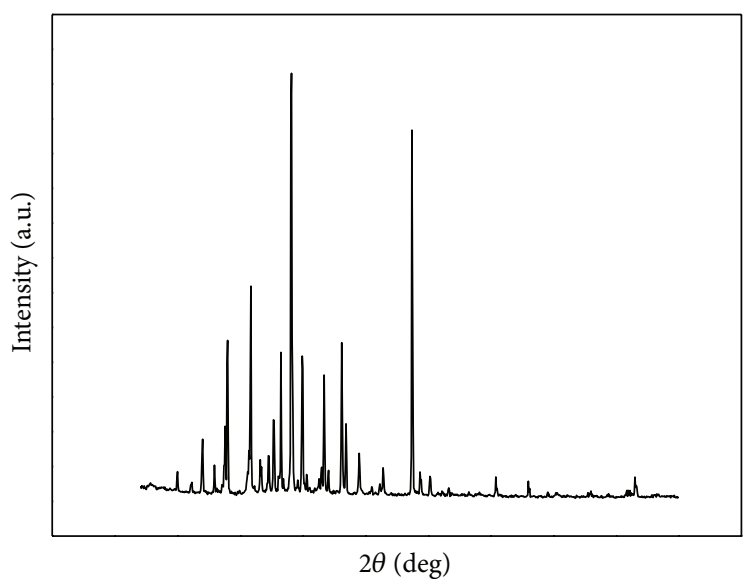

(c)

FIgURE 6: Powder XRD pattern of (a), TGSP (b), TGSP + ADP (c), and TGSP + KDP crystals.

TABLE 6: Electrical conductivity Analysis.

\begin{tabular}{lcccc}
\hline Crystal & $\begin{array}{c}\text { Electrical conductivity } \\
\text { Ec }(\text { Siemen } / \mathrm{cm})\end{array}$ & $\begin{array}{c}\text { Hopping frequency } \\
\omega_{p}(\mathrm{~Hz})\end{array}$ & $\begin{array}{c}\text { Charge carrier } \\
\text { concentration N/cm }\end{array}$ & Mobility $\mu\left(\mathrm{cm}^{2} / \mathrm{Vs}\right)$ \\
\hline TGSP & $1.3048 e-6$ & 12690.11 & $0.3290 e-8$ & $24.787 e+20$ \\
TGSP + ADP & $2.5054 e-6$ & 27080.365 & $0.2961 e-8$ & $52.8833 e+20$ \\
TGSP + KDP & $8.5078 e-6$ & 102880.996 & $0.2646 e-8$ & $200.95899 e+20$ \\
\hline
\end{tabular}

3.3. FT-Raman Spectral Analysis. Figure 5 shows FT-Raman spectra of pure and doped TGSP crystals. FT-Raman spectrum of pure TGSP matches very well with the earlier reported values of pure TGS $[2,17]$. Obtained Raman bands and their assignments are tabulated in Table 3. Raman band observed at $1414 \mathrm{~cm}^{-1}$ and $1605 \mathrm{~cm}^{-1}$ corresponding to symmetric and asymmetric stretching vibration of $\mathrm{COO}^{-}$group confirms the zwitterion configuration of glycine $[2,17]$. The band at $1683 \mathrm{~cm}^{-1}$ corresponding to symmetric vibration of $\mathrm{C}=\mathrm{O}$ group confirms the presence of glycinium ion configuration [17]. In FT-Raman spectra of ADP- and KDPdoped samples, some peaks were shifted to a considerable range compared to pure TGS. A change in intensity of all peaks was observed. The amount of polarisability change will determine the Raman scattering intensity. So, it can be concluded that the change in intensity of peaks may be due to incorporation of dopants.

3.4. Powder XRD Study. Figure 6 shows powder XRD pattern of pure and doped TGSP crystals. All crystals belong to monoclinic structure. XRD pattern of grown crystals differ from each other in intensity of reflection. Sharp and high intensity of reflection planes revealed that all crystals have good crystalline nature. Changes in Intensity of peaks for doped samples compared to pure TGSP revealed that there must be some changes in electron density in reflection planes 


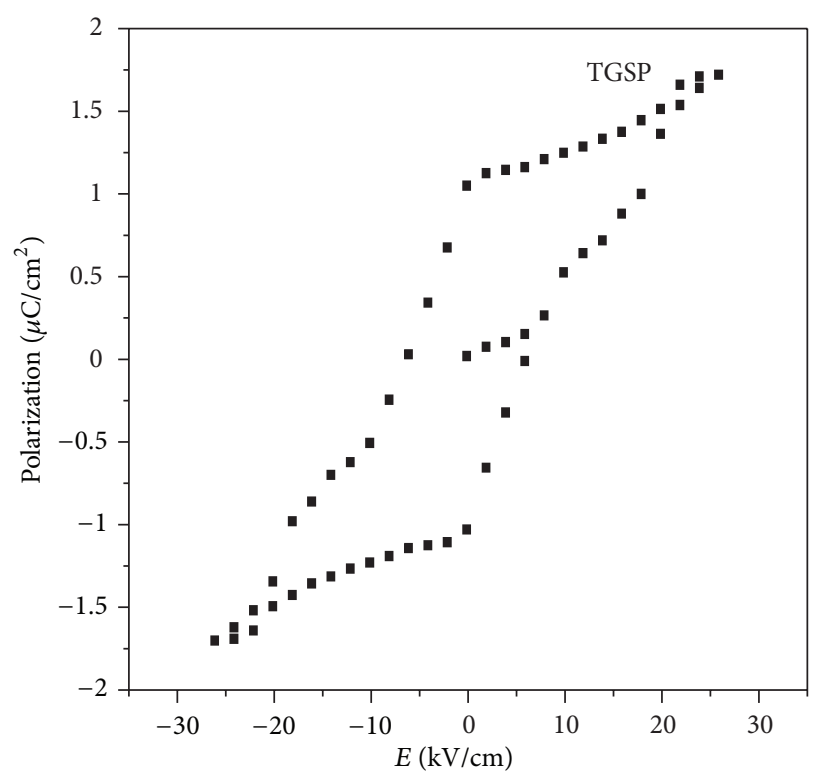

(a)

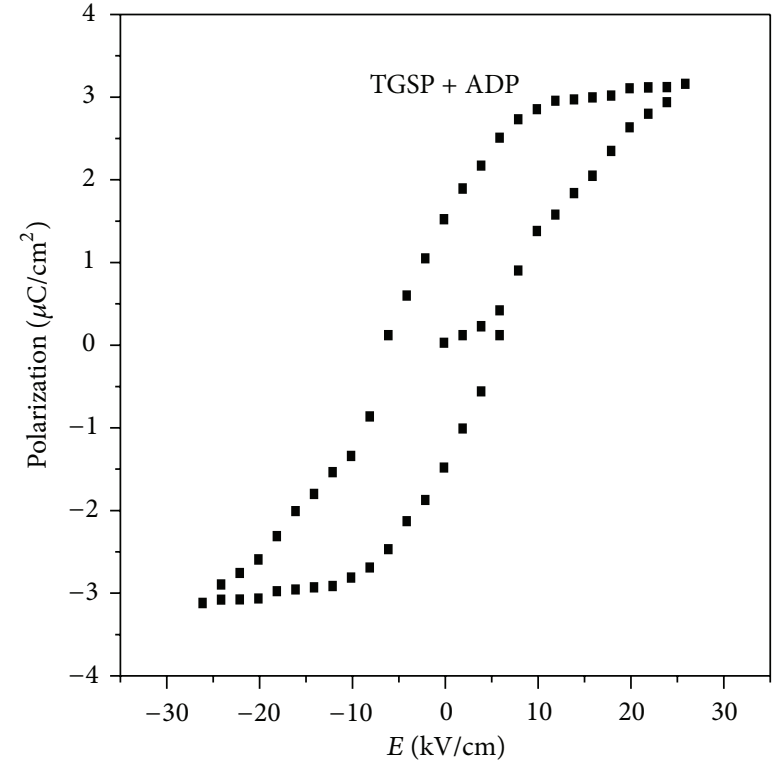

(b)

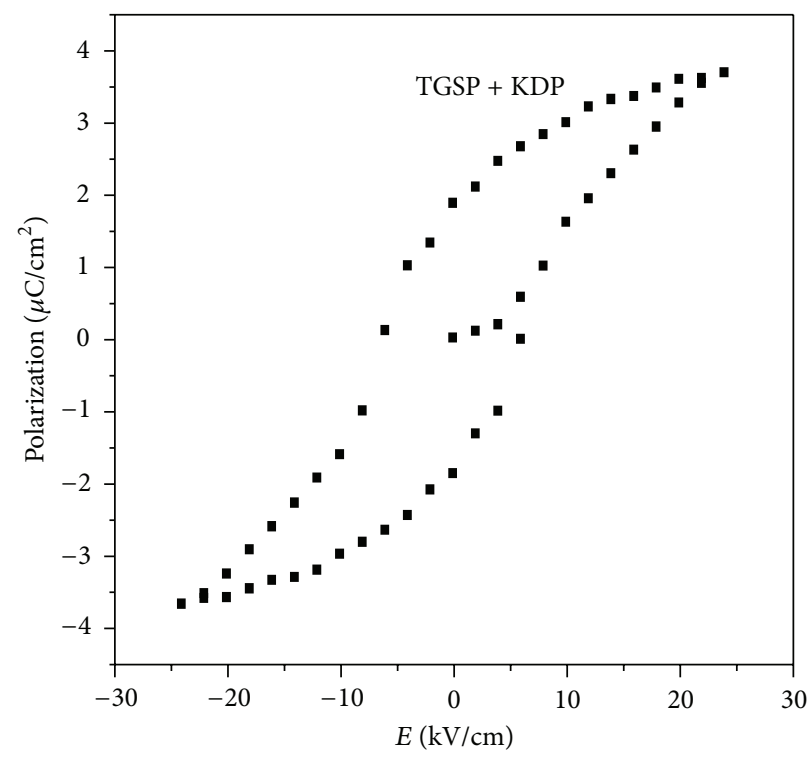

(c)

FIGURE 7: Ferroelectric hysteresis loops of (a) TGSP, (b) TGSP + ADP, and (c) TGSP + KDP crystals.

due to the incorporation of dopants. Small change in $2 \theta$ value is observed for doped samples. So, pure and doped samples have different morphologies. Lattice parameter values are shown in Table 4. Obtained lattice parameter values for pure and doped crystals slightly differ from each other. Because of doping, there may be some defects or strains in grown crystals.

3.5. Ferroelectric Hysteresis Study. Homemade Sawyer-Tower circuit is constructed [27]. Sample capacitors were prepared by using aluminium foils and the pure and doped TGSP samples as dielectric in between the aluminium foils [29]. Spontaneous polarization values $\left(P_{s}\right)$ for all samples were obtained by using the equation $P=Q / A$ micro coulomb $/ \mathrm{cm}^{2}$. Here, $Q$ is charge measured on sample capacitor (coulomb) and $A$ is area of capacitor plate $\left(\mathrm{cm}^{2}\right)$ [29]. Results are shown in Table 5. Figure 7 shows obtained ferroelectric hysteresis loops of grown crystals.

3.6. Electrical Measurement. Grown crystals are subjected to electrical characterization using IMPEDENCE ANALYSER IM3570. All crystals conduct electricity linearly. Doped crystals have higher electrical conductivity than pure TGSP crystal. Results are shown in Table 6. Figure 8 shows electrical conductivity graphs of grown crystals. Electrical conductivity graphs of pure and doped TGSP crystals contain two 


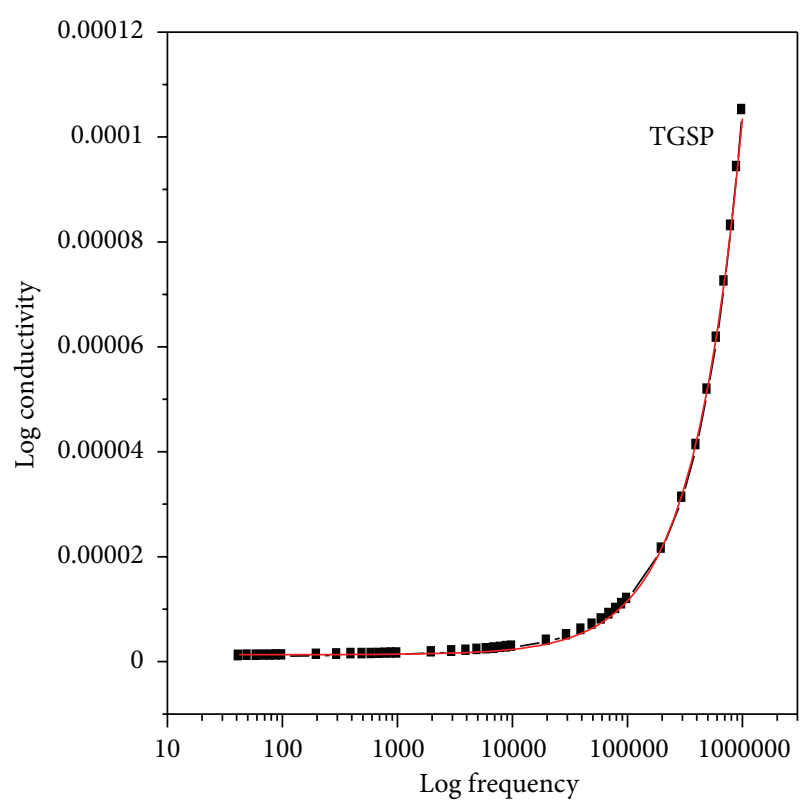

(a)

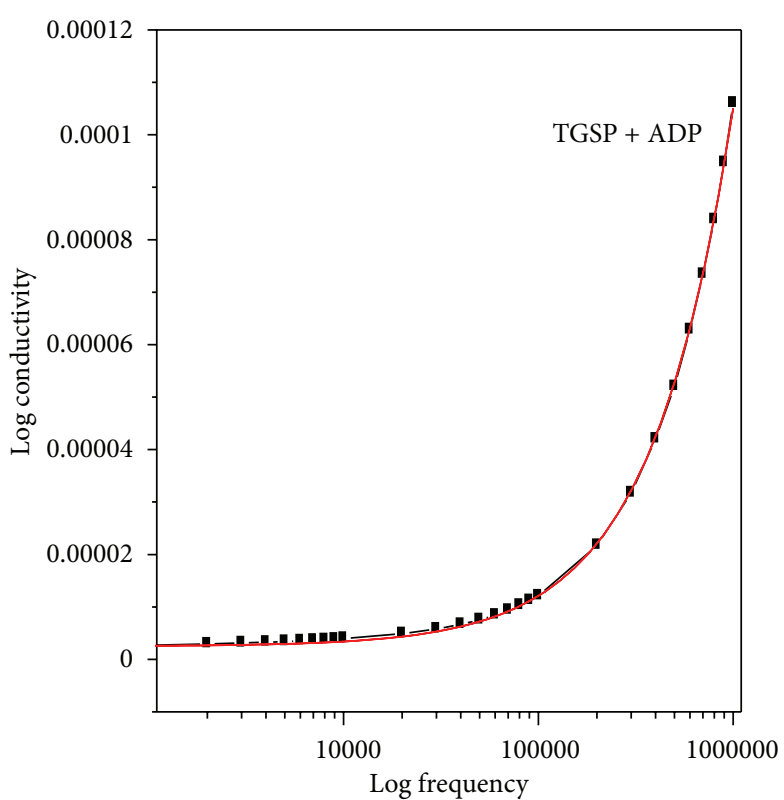

(b)

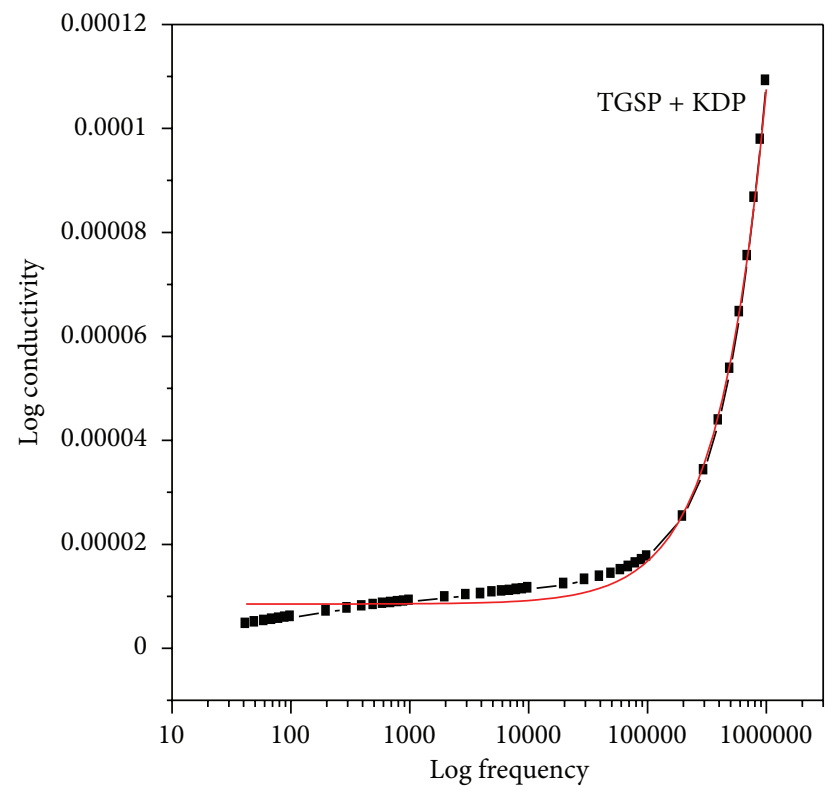

(c)

FIGURE 8: Frequency versus conductivity graphs of (a) TGSP, (b) TGSP + ADP, and (c) TGSP + KDP crystals.

regions: frequency-independent Ac conductivity region in low-frequency range and frequency-dependent dc conductivity region in high-frequency range. Conductivity graphs obey Arrhenius' relation and Jonscher's power law [30-33]. For all grown crystals, dc conductivity linearly increases with an increase in frequency. This indicates that electrical conductivity of these crystals is due to hopping mechanism. Electrical conductivity $\sigma_{\mathrm{dc}}$ is obtained by nonlinear fitting for the conductivity graphs. Then, hopping frequency $\omega_{p}$ is obtained by using the relation $\omega_{p}=\left(\sigma_{\mathrm{dc}} / A\right)^{1 / n}$. Here, $n$ is frequency exponent. $A$ is temperature-dependent parameter. Charge carrier concentration is obtained by $N=\sigma_{\mathrm{dc}} T / \omega_{p}$. Mobility of charge carriers is obtained by $\mu=\sigma_{\mathrm{dc}} / \mathrm{Ne}$.
Here, $e$ is charge of electron [32]. From electrical conductivity analysis, TGSP + KDP crystal has maximum dc electrical conductivity. It has higher hopping frequency value. Though TGSP + KDP has less charge carrier concentration compared to pure TGSP, it has higher value of mobility of charge carrier. This may contribute to the increase of dc electrical conductivity for TGSP + KDP crystal.

\section{Conclusion}

From UV-Vis spectra of grown crystals, it is confirmed that all these crystals have excellent optical quality. This property makes these crystals useful for applications in lasers, 
holographic recording, optical filters, and nonlinear optical applications and electrooptic applications. From FT-IR and FT-Raman spectral investigations, molecular structures of pure and doped TGSP crystals are verified. Less resolution of peaks and change in intensity of peaks for doped samples compared to pure TGSP are due to interaction between parent and dopants. It is concluded that ADP and KDP were well incorporated into the lattice of TGSP crystal. From powder XRD pattern, it is confirmed that all crystals are crystallizes in monoclinic structure. Ferroelectric hysteresis study reveals that for ADP- and KDP-doped samples spontaneous polarization values were slightly increased. So doped crystals have improved ferroelectric behavior than pure TGSP. So it can be concluded that ADP- and KDP-doped TGSP crystals are most suitable for infrared detector applications. From electrical conductivity measurements, it is observed that all crystals conduct electricity linearly and doped crystals have higher electrical conductivity than pure TGSP crystal.

\section{Acknowledgments}

The authors are thankful to the Management of Karpagam University for providing the facilities for the work, and to the Head, Department of Nanoscience and Technology, Bharathiar University, for recording the Raman spectra. Also thanks are due to the faculty and research scholars of the Department of Physics, Karpagam University, Coimbatore, India, for their help during the course of the work.

\section{References}

[1] S. Hoshino, Y. Okaya, and R. Pepsinsky, "Crystal structure of the ferroelectric phase of (Glycine) $)_{3} \cdot \mathrm{H}_{2} \mathrm{SO}_{4}$," Physical Review, vol. 115, no. 2, pp. 323-330, 1959.

[2] E. M. Mihaylova and H. J. Byrne, "Raman studies of TGS doped with $\mathrm{N}_{d}$," Journal of Physics and Chemistry of Solids, vol. 61, no. 12, pp. 1919-1925, 2000.

[3] M. Kay and R. Kliengberg, "The crystal structure of triglycine sulfate," Ferroelectrics, vol. 5, no. 1, pp. 45-52, 1973.

[4] K. Meera, S. Aravazhi, P. Santhana Raghavan, and P. Ramasamy, "Growth and characterisation of L-tyrosine-doped TGS crystals," Journal of Crystal Growth, vol. 211, no. 1, pp. 220-224, 2000.

[5] H. V. Alexandru, C. Berbecaru, F. Stanculescu, L. Pintile, I. Matei, and M. Lisca, "Doped TGS crystals for IR detection and sensors," Sensors and Actuators A, vol. 113, no. 3, pp. 387-392, 2004.

[6] A. M. Malyarevich and M. R. Posledovich, "The assignment of lattice vibrations in triglycine sulfate-type crystals," Journal of Molecular Structure, vol. 375, no. 1-2, pp. 43-51, 1996.

[7] G. Arunmozhi, S. Lanceros-Méndez, and E. de Matos Gomes, "Antiferroelectric ADP doping in ferroelectric TGS crystals," Materials Letters, vol. 54, no. 5-6, pp. 329-336, 2002.

[8] S. Kalainathan, M. B. Margaret, and T. Irusan, "Morphological changes of L-asparagine doped TGS crystal," Crystal Engineering, vol. 5, no. 1, pp. 71-78, 2002.

[9] D. Jayalakshmi and J. Kumar, "Growth and characterization of 1tryptophan-doped ferroelectric TGS crystals," Journal of Crystal Growth, vol. 310, no. 7-9, pp. 1497-1500, 2008.

[10] K. Meera, R. Muralidharan, A. K. Tripathi, and P. Ramasamy, "Growth and characterisation of l-threonine, dl-threonine and 1-methionine admixtured TGS crystals," Journal of Crystal Growth, vol. 263, no. 1-4, pp. 524-531, 2004.

[11] A. Saxena, V. Gupta, and K. Sreenivas, "Characterization of phosphoric acid doped TGS single crystals," Journal of Crystal Growth, vol. 263, no. 1-4, pp. 192-202, 2004.

[12] W. Kulita and M. Trybus, "TGS single crystals doped with lysine: new material for IR detectors," SPIE Proceedings, vol. 5124, 2003.

[13] K. Meera, R. Muralidharan, P. Santhanaraghavan, R. Gopalakrishnan, and P. Ramasamy, "Growth and characterisation of Lcystine doped TGS crystals," Journal of Crystal Growth, vol. 226, no. 2-3, pp. 303-312, 2001.

[14] K. Meera, R. Muralidharan, A. K. Tripathi, R. Dhanasekaran, and P. Ramasamy, "Growth of thiourea-doped TGS crystals and their characterisation," Journal of Crystal Growth, vol. 260, no. 3-4, pp. 414-421, 2004.

[15] C. Berbecaru, H. V. Alexandru, L. Pintilie, A. Dutu, B. Logofatu, and R. C. Radulescu, "Doped versus pure TGS crystals," Materials Science and Engineering B, vol. 118, no. 1-3, pp. 141146, 2005.

[16] G. Su, Y. He, H. Yao, Z. Shi, and Q. Wu, "New pyroelectric crystal L-lysine-doped TGS (LLTGS)," Journal of Crystal Growth, vol. 209, no. 1, pp. 220-222, 2000.

[17] R. Parimaladevi, C. Sekar, and V. Krishnakumar, "The effect of nitric acid $\left(\mathrm{HNO}_{3}\right)$ on growth, spectral, thermal and dielectric properties of triglycine sulphate (TGS) crystal," Spectrochimica Acta A, vol. 75, no. 2, pp. 617-623, 2010.

[18] K. Meera, A. Claude, R. Muralidharan, C. K. Choi, and P. Ramasamy, "Growth and characterisation of EDTA-added TGS crystals," Journal of Crystal Growth, vol. 285, no. 3, pp. 358-364, 2005.

[19] A. Wojciechowski, I. V. Kityk, G. Lakshminarayana et al., "Laser-induced optical effects in triglycine-zinc chloride single crystals," Physica B, vol. 405, no. 13, pp. 2827-2830, 2010.

[20] V. Krishnakumar, S. Sivakumar, R. Nagalakshmi, S. Bhuvaneswari, and M. Rajaboopathi, "Effect of doping an organic molecule ligand on TGS single crystals," Spectrochimica Acta A, vol. 71, no. 2, pp. 480-485, 2008.

[21] T. Bharthasarathi, V. Siva Shankar, R. Jayavel, and P. Murugakoothan, "Growth and characterization of biadmixtured TGS single crystals," Journal of Crystal Growth, vol. 311, no. 4, pp. 1147-1151, 2009.

[22] R. Muralidharan, R. Mohankumar, P. M. Ushasree, R. Jayavel, and P. Ramasamy, "Effect of rare-earth dopants on the growth and properties of triglycine sulphate single crystals," Journal of Crystal Growth, vol. 234, no. 2-3, pp. 545-550, 2002.

[23] A. Abu El-Fadl, "Optical properties of TGS crystals doped with metal ions in the vicinity of phase transition," Physica B, vol. 269, no. 1, pp. 60-68, 1999.

[24] K. Ćwikiel, B. Fugiel, and M. Mierzwa, "Rigid domain structure in TGS ferroelectric," Physica B, vol. 293, no. 1-2, pp. 58-66, 2000.

[25] R. Mohan Kumar, R. Muralidharan, D. Rajan Babu et al., "Growth and characterization of L-lysine doped TGS and TGSP single crystals," Journal of Crystal Growth, vol. 229, no. 1, pp. 568-573, 2001.

[26] C. Rai, K. Sreenivas, and S. M. Dharmaprakash, "Improved ferroelectric and pyroelectric parameters in iminodiacetic acid doped TGS crystal," Journal of Crystal Growth, vol. 312, no. 2, pp. 273-275, 2010. 
[27] C. B. Sawyer and C. H. Tower, "Rochelle salt as a dielectric," Physical Review, vol. 35, no. 3, pp. 269-273, 1930.

[28] T. Balu, T. R. Rajasekaran, and P. Murugakoothan, "Studies on the growth, structural, optical and mechanical properties of ADP admixtured TGS crystals," Current Applied Physics, vol. 9, no. 2, pp. 435-440, 2009.

[29] M. Dawber, I. Farnan, and J. F. Scott, "A classroom experiment to demonstrate ferroelectric hysteresis," The American Journal of Physics, vol. 71, no. 8, pp. 819-822, 2003.

[30] C. S. Ramya, S. Selvasekarapandian, T. Savitha et al., "Conductivity and thermal behavior of proton conducting polymer electrolyte based on poly (N-vinyl pyrrolidone)," European Polymer Journal, vol. 42, no. 10, pp. 2672-2677, 2006.

[31] R. Baskaran, S. Selvasekarapandian, N. Kuwata, J. Kawamura, and T. Hattori, "Conductivity and thermal studies of blend polymer electrolytes based on PVAc-PMMA," Solid State Ionics, vol. 177, no. 26-32, pp. 2679-2682, 2006.

[32] V. D. Nithya, R. Jacob Immanuel, S. T. Senthilkumar et al., "Studies on the structural, electrical and magnetic properties of $\mathrm{LaCrO}_{3}, \mathrm{LaCr}_{0.5} \mathrm{Cu}_{0.5} \mathrm{O}_{3}$ and $\mathrm{LaCr}_{0.5} \mathrm{Fe}_{0.5} \mathrm{O}_{3}$ by sol-gel method," Materials Research Bulletin, vol. 47, no. 8, pp. 1861-1868, 2012.

[33] V. D. Nithya and R. Kalai Selvan, "Synthesis, electrical and dielectric properties of $\mathrm{FeVO}_{4}$ nanoparticles," Physica B, vol. 406, no. 1, pp. 24-29, 2011. 

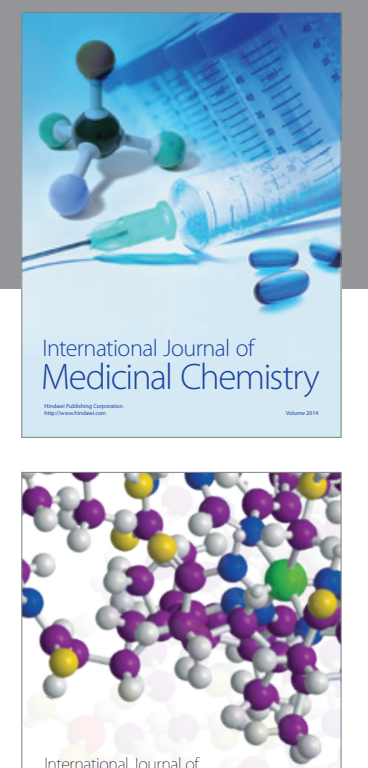

\section{Carbohydrate} Chemistry

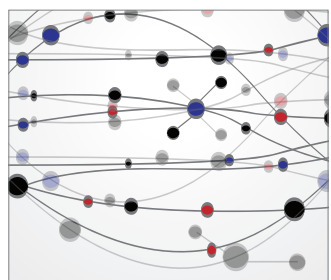

The Scientific World Journal
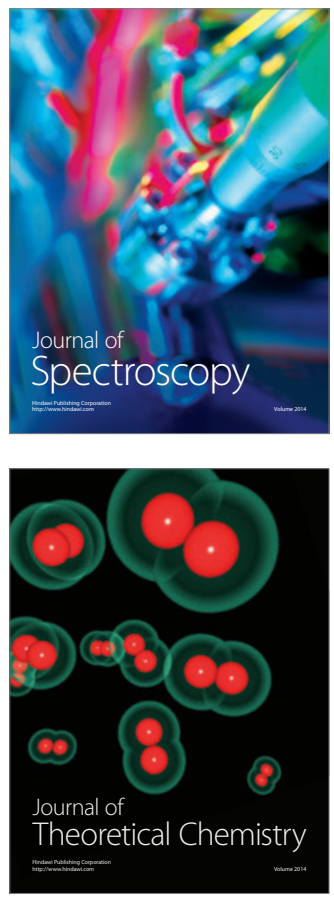
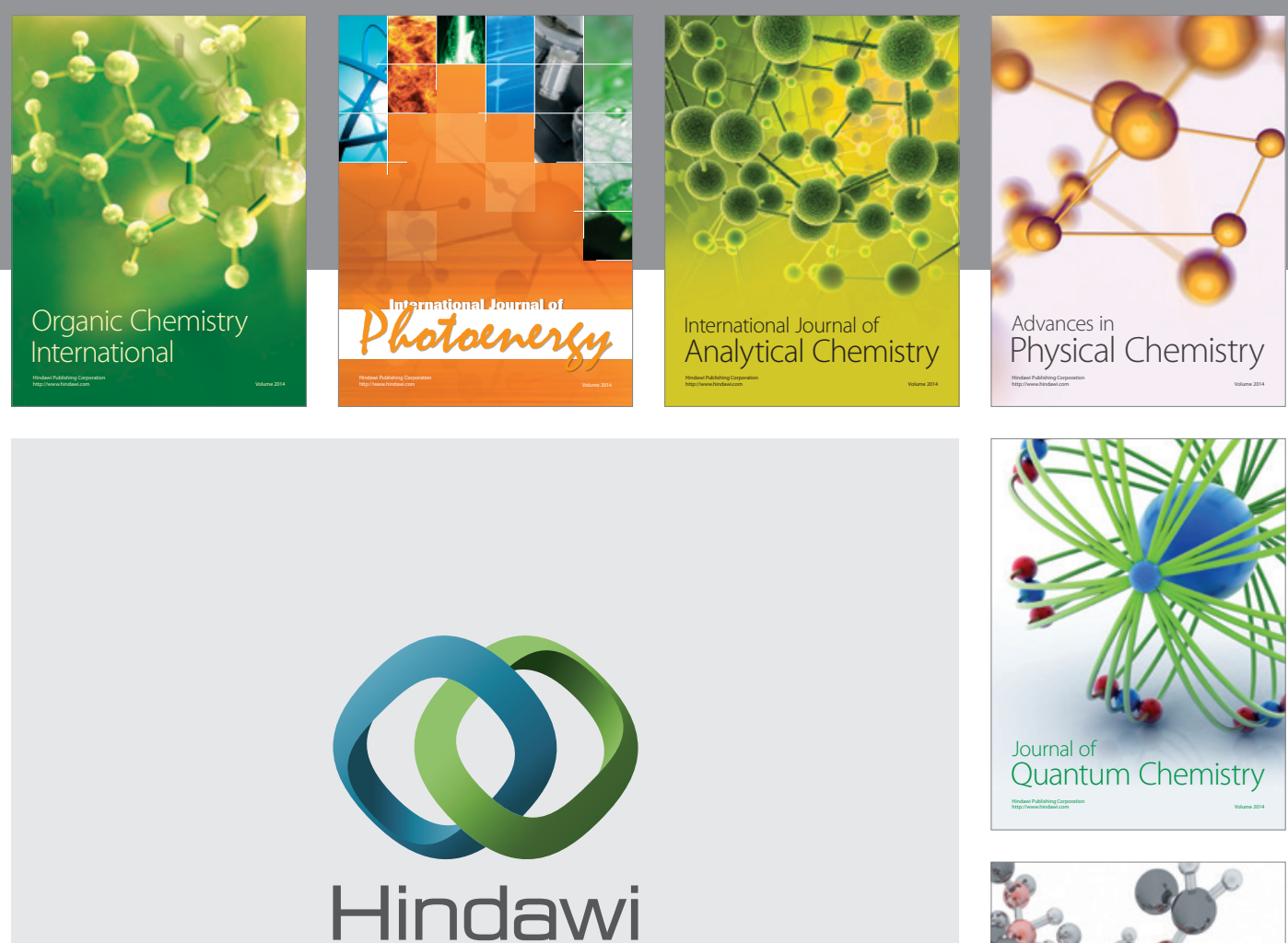

Submit your manuscripts at

http://www.hindawi.com

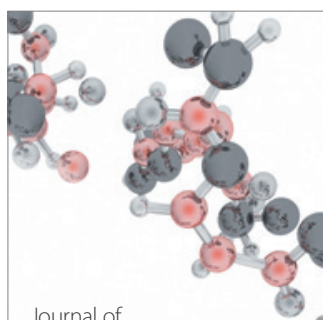

Analytical Methods

in Chemistry

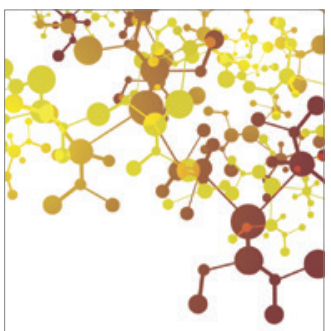

Journal of

Applied Chemistry

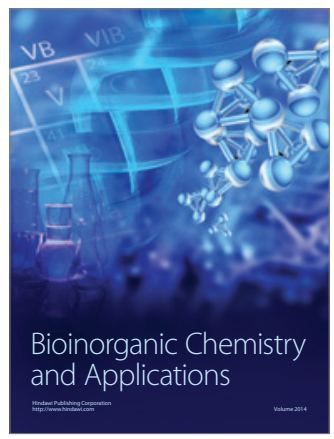

Inorganic Chemistry
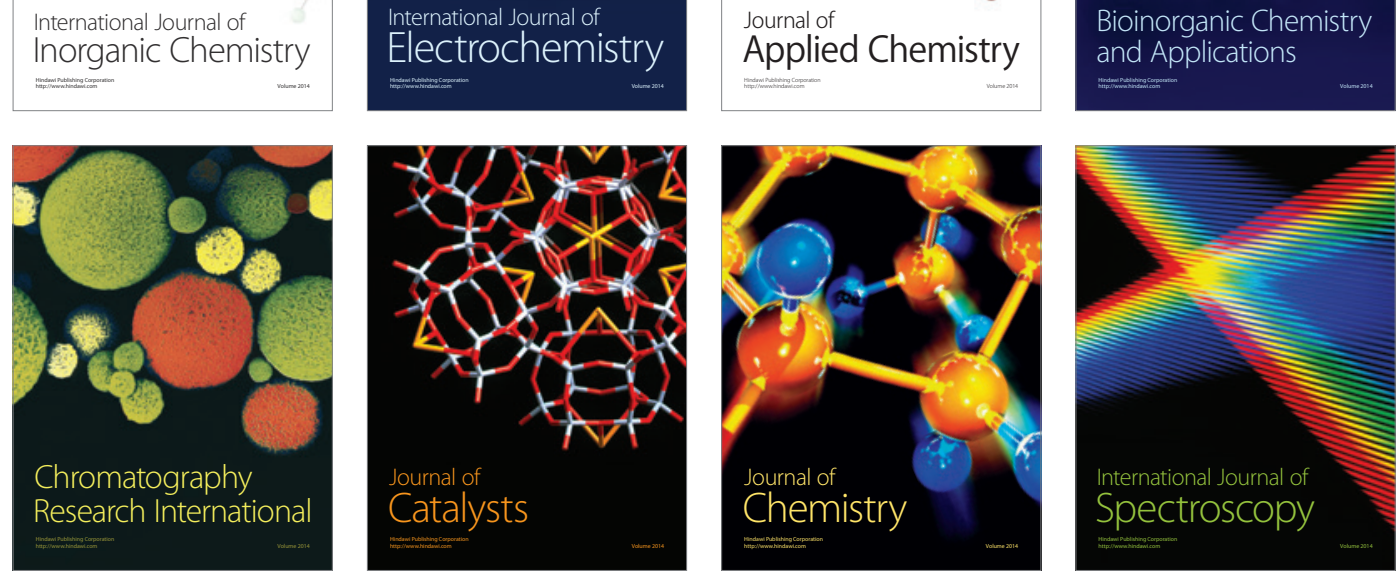